\title{
The Associations between Paraoxonase 1 L55M/Q192R Genetic Polymorphisms and the Susceptibilities of Diabetic Macroangiopathy and Diabetic Microangiopathy: A Meta-Analysis
}

\author{
Chenfang $\mathrm{Wu} \cdot$ Diling $\mathrm{Wu} \cdot$ Minjie Lin $\cdot$ Yanjun Zhong (D)
}

Received: May 11, 2018 / Published online: July 9, 2018

(c) The Author(s) 2018

\section{ABSTRACT}

Introduction: Plenty of studies have focused on the associations of paraoxonase 1 Q192R and L55M genetic polymorphisms with diabetic macroangiopathy and microangiopathy susceptibility, but these associations remain controversial. Therefore, this meta-analysis was conducted to demonstrate these relationships. Methods: Relevant studies published in English or Chinese were identified in PubMed, Embase, Wanfang Database, and CNKI by applying specific inclusion and exclusion criteria. Statistical analyses were performed using the STATA 12.0 statistical software.

Results: 25 Case-control studies were included in the meta-analyses: six on the association

Enhanced digital features To view enhanced digital features for this article go to https://doi.org/10.6084/ m9.figshare.6586889.

Electronic supplementary material The online version of this article (https://doi.org/10.1007/s13300018-0466-5) contains supplementary material, which is available to authorized users.

C. Wu $\cdot$ D. Wu $\cdot$ Y. Zhong (ه)

ICU Center, The Second Xiangya Hospital, Central South University, Furong, Changsha, China

e-mail: zhongyanjun@csu.edu.cn

M. Lin

Clinical Skills Training Center, The Second Xiangya

Hospital, Central South University, Furong,

Changsha, China between paraoxonase 1 L55M genetic polymorphism and diabetic macroangiopathy risk, nine on the association between $\mathrm{L} 55 \mathrm{M}$ and diabetic microangiopathy risk, 12 on the association between Q192R and diabetic macroangiopathy risk, and 12 on the association between Q192R and diabetic microangiopathy risk. Paraoxonase 1 L55M genetic polymorphism was significantly associated with diabetic microangiopathy susceptibility in the dominant model [odds ratio (OR) $0.53,95 \%$ confidence interval (CI) $0.33-0.83, \quad P=0.006]$, the homozygous model (OR 0.37, 95\% CI $0.16-0.86, P=0.021)$, the allelic contrast model (OR $0.62,95 \%$ CI $0.43-0.90, P=0.011$ ), the recessive model (OR 12.04, 95\% CI 8.02-18.06, $P=0.000$ ), and the heterozygous model (OR $0.57,95 \%$ CI $0.38-0.85, P=0.006$ ), but L55M was not significantly associated with macroangiopathy susceptibility. Paraoxonase 1 Q192R genetic polymorphism was significantly associated with diabetic macroangiopathy susceptibility in the homozygous model (OR 1.88, 95\% CI 1.06-3.32, $P=0.030$ ), the allelic contrast model (OR 1.31, 95\% CI 1.02-1.69, $P=0.038$ ), and the recessive model (OR 1.55, 95\% CI $1.11-2.16, P=0.010)$, but not in the dominant and heterozygous models. Meanwhile, there was no significant association between paraoxonase 1 Q192R genetic polymorphism and diabetic microangiopathy susceptibility.

Conclusion: Paraoxonase 1 L55M and Q192R genetic polymorphisms play important roles in 
diabetic macroangiopathy and microangiopathy susceptibility. Further well-designed studies based on large samples are needed to confirm these results.

Keywords: Diabetic macroangiopathy; Diabetic microangiopathy; Paraoxonase 1; Polymorphism

\section{INTRODUCTION}

Diabetes mellitus (DM) is a highly investigated and complex chronic disease that has become increasingly prevalent with economic development. In 2016, DM was reported to be the eighth most prevalent cause of disease-related mortality. If DM is not properly managed, it can result in diabetic macroangiopathy (of the heart, brain, lower limb arteries, etc.), a specific form of accelerated atherosclerosis, as well as diabetic microangiopathy (of the kidney or eye, neuropathy, etc.), which are associated with increased morbidity and mortality. It is reported that $20-30 \%$ of diabetic patients have diabetic macroangiopathy [1]. Atherosclerosis in DM is more severe and aggressive than that in nonDM patients, and usually involves multiple arteries. DM also adversely affects the microvasculature in many organs, and remains the leading cause of chronic kidney disease and blindness [2]. Therefore, the ultimate goal of managing DM is to lower the risks of diabetic macroangiopathy (DMMA) and highly morbid microangiopathy (DMMI).

There are many risk factors associated with diabetic macroangiopathy and microangiopathy, including genetic factors, dyslipidemia, smoking, alcohol, obesity, exercise, and oxidative stress, but diabetic macroangiopathy and microangiopathy susceptibility is still not completely understood. Paraoxonase 1 is a 354-aa, $45-\mathrm{kDa}$ glycoprotein that is synthesized in the liver and released into the blood, and which binds to high-density lipoprotein in a calciumdependent manner [3]. Paraoxonase 1 is the main constituent of high-density lipoprotein (HDL), and contributes to the protective role of HDL against vascular diseases [4]. Paraoxonase 1 activity is reported to affect diabetic macroangiopathy and microangiopathy susceptibility [4-6]. There are large ethnic differences in the genetic polymorphisms of paraoxonase 1 , according to the 1000 Genomes database [7]. A meta-analysis showed that paraoxonase 1 L55M and Q192R genetic polymorphisms were significantly associated with susceptibility to DM, but there were notable ethnic differences [7]. In 1998, Kao et al. reported that paraoxonase 1 L55M genetic polymorphism was significantly associated with diabetic retinopathy, whereas Q192R was not [8]. In another study, the author found that both (i.e., paraoxonase 1 L55M and Q192R) genetic polymorphisms were significantly related to diabetic complications [9]. Plenty of studies have focused on the associations of paraoxonase 1 Q192R and L55M genetic polymorphisms with diabetic macroangiopathy and microangiopathy susceptibility, but these links remain controversial. Therefore, the metaanalysis reported in the present paper was conducted to demonstrate these relationships.

\section{METHODS}

\section{Search Strategy}

We carried out literature searches for papers published in English or Chinese in the Embase, PubMed, Wanfang Database, and China National Knowledge Internet (CNKI) databases using the following keywords: "PON1" or "paraoxonase 1," "diabetes" or "diabetes mellitus" or "DM" or "Type 2 diabetes mellitus (T2DM)" or "Type 1 diabetes mellitus (T1DM)," and "variation" or "polymorphism" or "single nucleotide polymorphism" or "SNP." We also searched through relevant articles manually to find additional reports.

\section{Inclusion and Exclusion Criteria}

Articles were included in this meta-analysis if they complied with the following criteria: (1) they were published as original case-control studies; (2) they reported the relationship(s) of paraoxonase 1 L55M and/or Q192R genetic 
polymorphisms with diabetic macroangiopathy and/or diabetic microangiopathy susceptibility; (3) they used DM without any complication as a control. When the same series of patients were used in more than one article, we used the latest and most complete study.

\section{Data Extraction}

WCF and WDL screened all of the articles found in the searches and extracted data from all eligible publications independently. For each study included in our meta-analysis, we carefully extracted the following information: first author, published year, country, ethnicity, type of DM, diagnostic criteria used for diabetic complications, and paraoxonase $1 \mathrm{~L} 55 \mathrm{M}$ and/or Q192R genotypes in each group. If there were any discrepancies between the data extracted by WCF and WDL, they attempted to resolve the disagreement through discussion; if no agreement was reached, another author was consulted to resolve the dispute.

\section{Compliance with Ethics Guidelines}

This article is based on previously conducted studies and does not contain any studies with human participants or animals performed by any of the authors.

\section{Statistical Analysis}

Our meta-analysis followed the recommendations of the PRISMA statement and used the Newcastle-Ottawa Scale (NOS) criteria to assess study quality. Studies that met five or more of the NOS criteria were considered of high quality. Pooled odds ratios (ORs) were used to explore the relationships of paraoxonase 1 L55M and Q192R polymorphisms with the susceptibilities to diabetic macroangiopathy and/or diabetic microangiopathy. Heterogeneity was measured using the chi-square-based $Q$ test. A random effects model was applied when $I^{2}>50 \%$ and $P<0.05$. Otherwise, a fixed effects model was used. Publication bias was investigated using Egger's test and a funnel plot. This meta-analysis was performed using STATA 12.0 software (Stata Corporation, TX, USA). $P<0.05$ was taken to imply statistical significance.

\section{RESULTS}

\section{Study Characteristics}

As shown in Fig. S1 of the Electronic supplementary material (ESM), 332 publications were identified, among which 111 duplicates and 57 irrelevant papers were subsequently excluded. Of the remaining 164 papers, 139 were excluded for the following reasons: the paper was a review, no diabetic macroangiopathy or microangiopathy groups were included, there was no DM without complication group, the paper was a duplicate, and other paraoxonase 1 genetic polymorphisms were studied. Thus, 25 articles were ultimately included in the present meta-analysis. Eleven publications were written in English [8-18] and 14 were written in Chinese [19-32]. Table 1 shows detailed information on those 25 studies. The name of the first author, the year published, country, ethnicity, the type of DM, the numbers and ages of the cases and controls, the diagnostic criteria used for diabetic complications, and the genetic polymorphisms of paraoxonase 1 studied in each work are presented. All studies were found to be of high quality according to the NOS criteria.

\section{Paraoxonase 1 L55M Genetic Polymorphism and Risk of Diabetic Macroangiopathy}

As shown in Table 2, six studies probed the association between paraoxonase 1 L55M genetic polymorphism and the risk of diabetic macroangiopathy; these studies included 431 cases and 640 DM patients without complications as the control. Two studies were performed in Europe [10, 17], and four were carried out in Asia [9, 16, 31, 32]. The results indicated that there was no heterogeneity in the dominant model (LM $+\mathrm{MM}$ vs $\mathrm{LL}: I^{2}=0.0 \%$, $P=0.447$, Fig. 1a, Table 4), homozygous model (MM vs LL: $I^{2}=30.7 \%, \quad P=0.217$, Fig. $1 \mathrm{~b}$, 


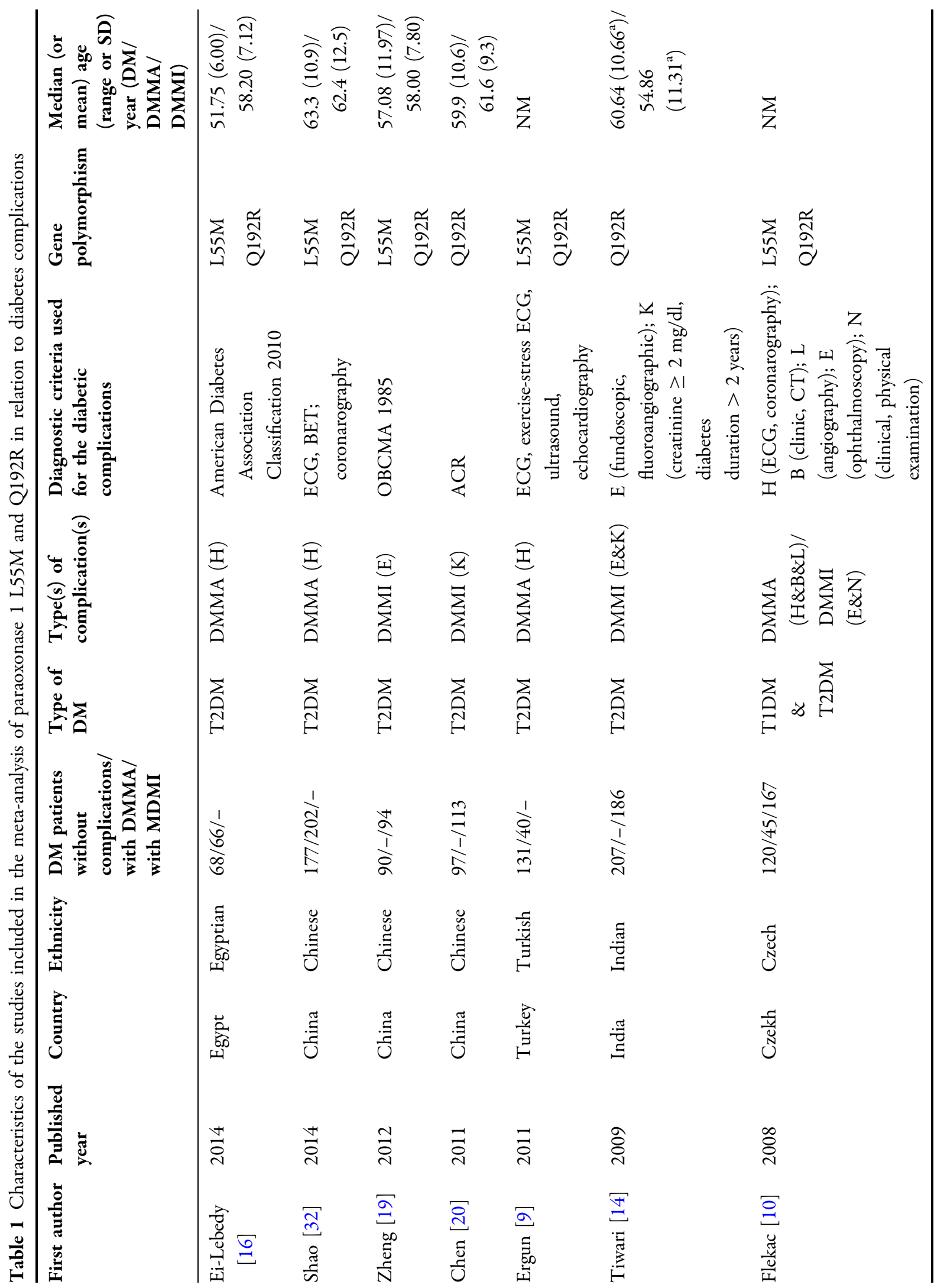




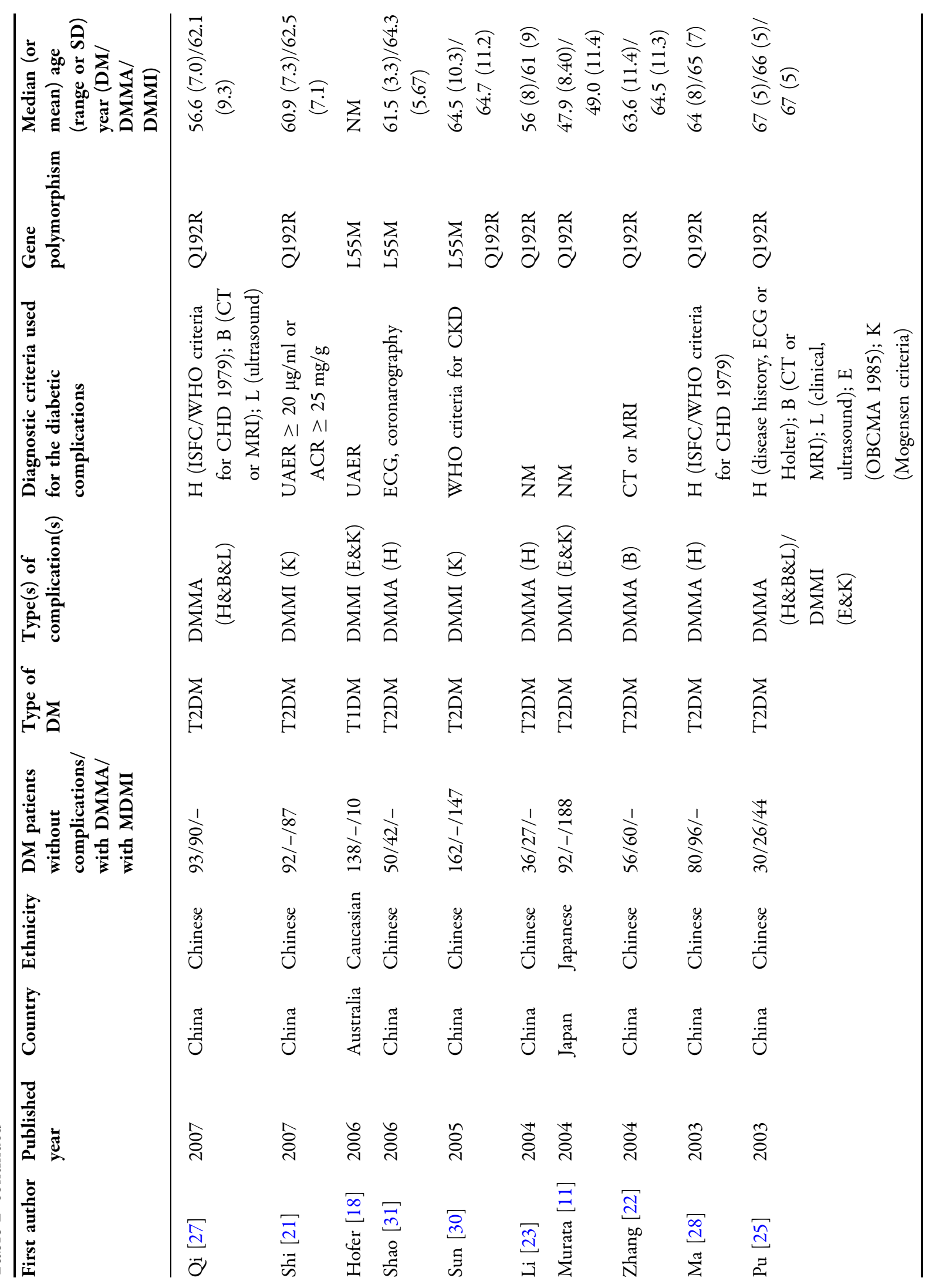




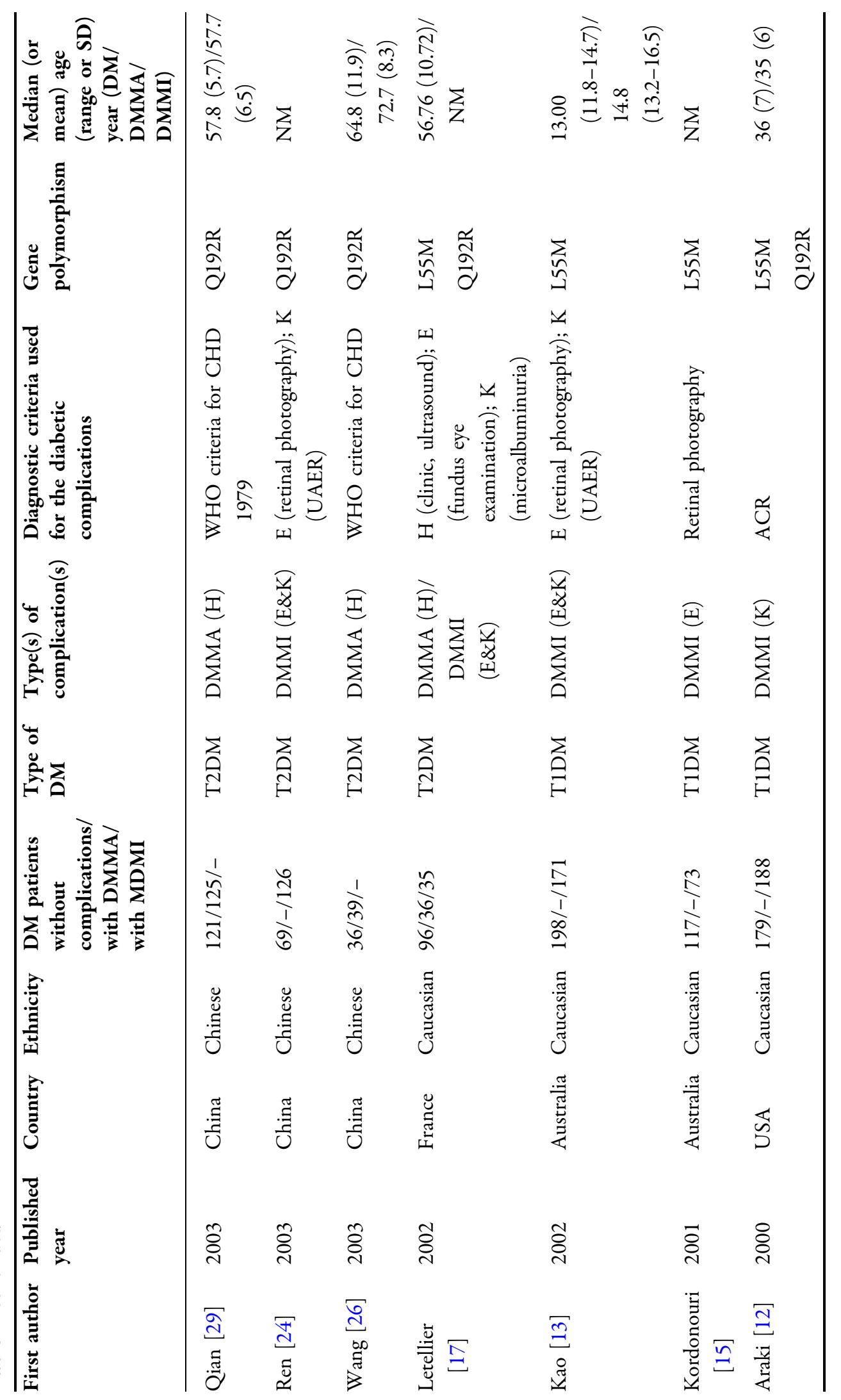




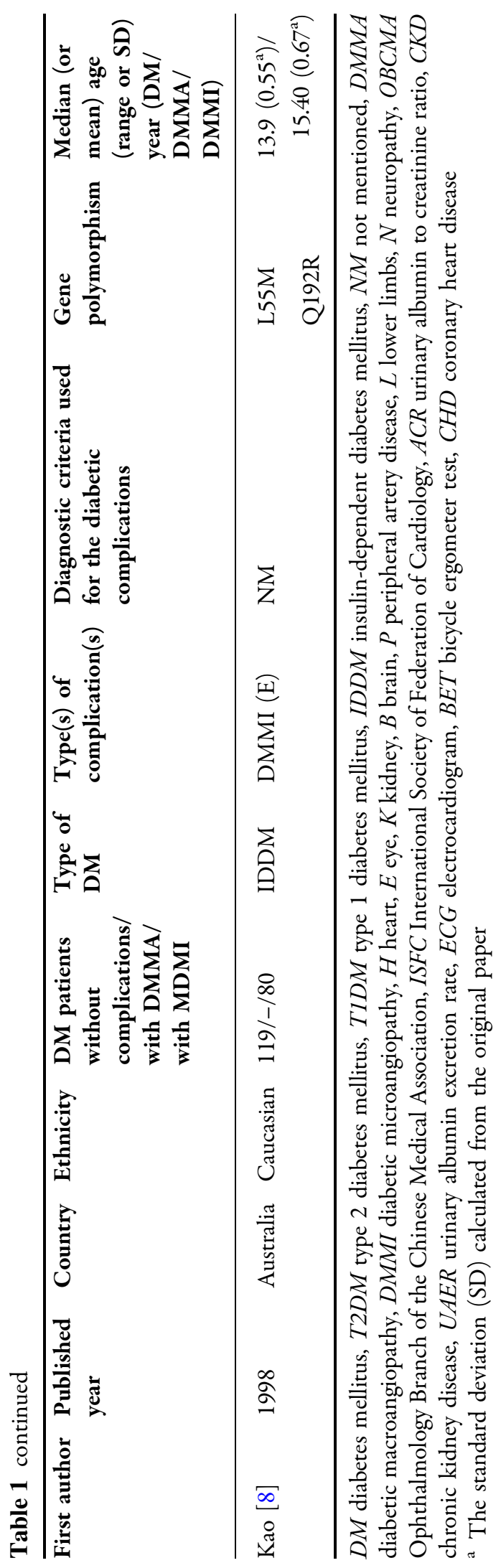

Table 4), allelic contrast model (M vs L: $I^{2}=16.8 \%, P=0.305$, Fig. $1 \mathrm{c}$, Table 4$)$, recessive model (MM vs LL + LM: $I^{2}=19.9 \%, P=0.288$, Fig. 1d, Table 4), and heterozygous model (LM vs LL: $I^{2}=0.0 \%, P=0.610$, Fig. $1 \mathrm{e}$, Table 4 ). Meta-analysis suggested that there was no significant association of paraoxonase 1 L55M genetic polymorphism with susceptibility to diabetic macroangiopathy in all five models (LM + MM vs LL: OR 0.98, 95\% CI 0.69-1.38, $P=0.996$, Fig. 1a; MM vs LL: OR $1.30,95 \%$ CI $0.81-2.08, P=0.279$, Fig. $1 \mathrm{~b}$; and $M$ vs $L$ : OR 1.10, 95\% CI $0.87-1.39, P=0.414$, Fig. $1 c ; M M$ vs LLLM: OR 1.30, 95\% CI 0.99-1.90, $P=0.176$, Fig. 1d; LM vs LL: OR 0.90, 95\% CI 0.62-1.30, $P=0.569$, Fig. 1e; Table 4). When the effects models were altered, the significances of these three models did not change statistically significantly (data not shown). Based on the funnel plot and Egger's test, none of the three models had significant publication bias (LM + MM vs LL: $t=-0.040, P=0.970 ; \mathrm{MM}$ vs LL: $t=0.300, \quad P=0.781 ; \quad \mathrm{M} \quad$ vs $\mathrm{L}: \quad t=-0.340$, $P=0.752 ; \mathrm{MM}$ vs LL + LM: $t=0.69, P=0.539$; LM vs LL: $t=-0.10, P=0.923$; data not shown).

\section{Paraoxonase 1 L55M Genetic Polymorphism and Susceptibility to Diabetic Microangiopathy}

As shown in Table 2, nine studies focused on the association of paraoxonase 1 L55M genetic polymorphism with the risk of diabetic microangiopathy; these studies included 992 cases and 1212 controls $[8,10,12,13,15$, $17-19,30]$. The results showed that there was significant heterogeneity in the dominant model (LM + MM vs LL: $I^{2}=81.0 \%, P=0.000$, Fig. 2a, Table 4), homozygous model (MM vs LL: $I^{2}=80.9 \%, P=0.000$, Fig. $2 b$, Table 4$)$, allelic contrast model (M vs L: $I^{2}=84.1 \%, P=0.000$, Fig. 2c, Table 4), recessive model (MM vs LL + LM: $I^{2}=68.6 \%, P=0.001$, Fig. 2 d, Table 4$)$, and heterozygous model (LM vs LL: $I^{2}=71.1 \%$, $P=0.000$, Fig. 2e, Table 4$)$. Thus, subgroup analysis of ethnicity, type of DM, and Hardy-Weinberg equilibrium (HWE) was performed, and the results indicated that ethnicity 


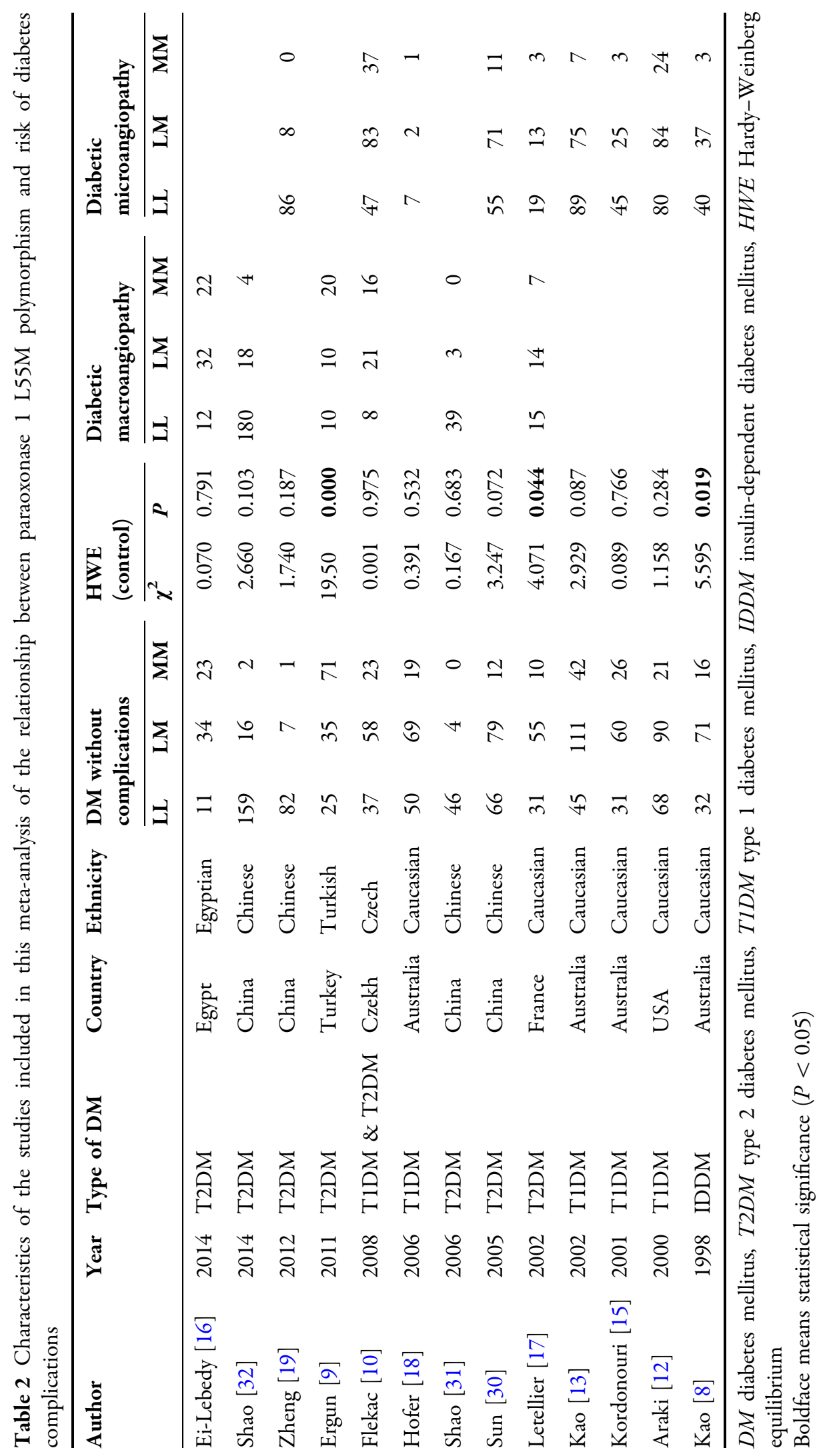




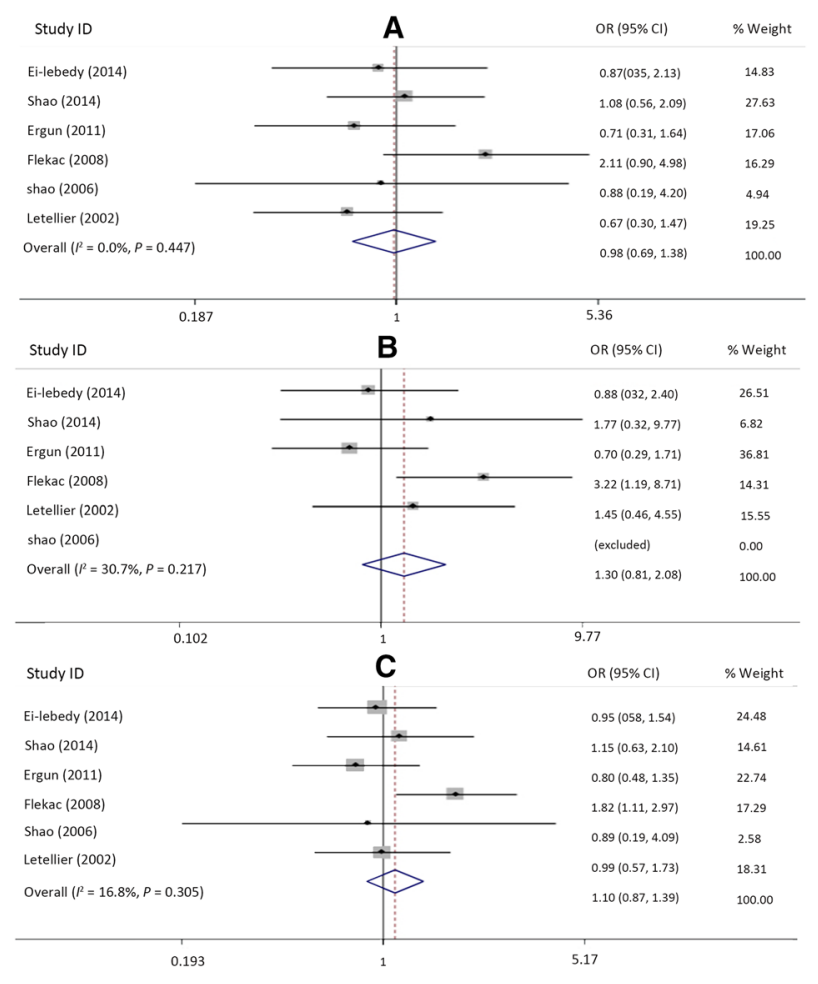

Fig. 1a-e Forest plots for the association of paraoxonase 1 L55M genetic polymorphism with diabetic macroangiopathy. a Dominant model, LM + MM vs LL; $\mathbf{b}$ homozygous

and type of DM can explain the heterogeneity in the recessive model, but not the heterogeneity in the other models (Table 4). Meta-regression analysis was then performed using the covariates published year, sample size, ethnicity, type of DM, and HWE to investigate the sources of the between-study heterogeneity, but it failed to identify those sources (Table 5). Paraoxonase $1 \mathrm{~L} 55 \mathrm{M}$ genetic polymorphism was clearly related to diabetic microangiopathy susceptibility in the dominant model (OR 0.53, 95\% CI 0.33-0.83, $P=0.006$, Fig. 2a, Table 4), the homozygous model (OR 0.37, 95\% CI $0.16-0.86, P=0.021$, Fig. $2 b$, Table 4 ), the allelic contrast model (OR $0.62,95 \%$ CI 0.43-0.90, $P=0.011$, Fig. 2c, Table 4), the recessive model (OR 12.04, 95\% CI 8.02-18.06, $P=0.000$, Fig. 2d, Table 4), and the heterozygous model (OR 0.57, 95\% CI 0.38-0.85, $P=0.006$, Fig. 2e, Table 4). When the effects models were changed, the statistical significance did not alter (data not shown). Meanwhile, the funnel plot and Egger's test indicated that there was no

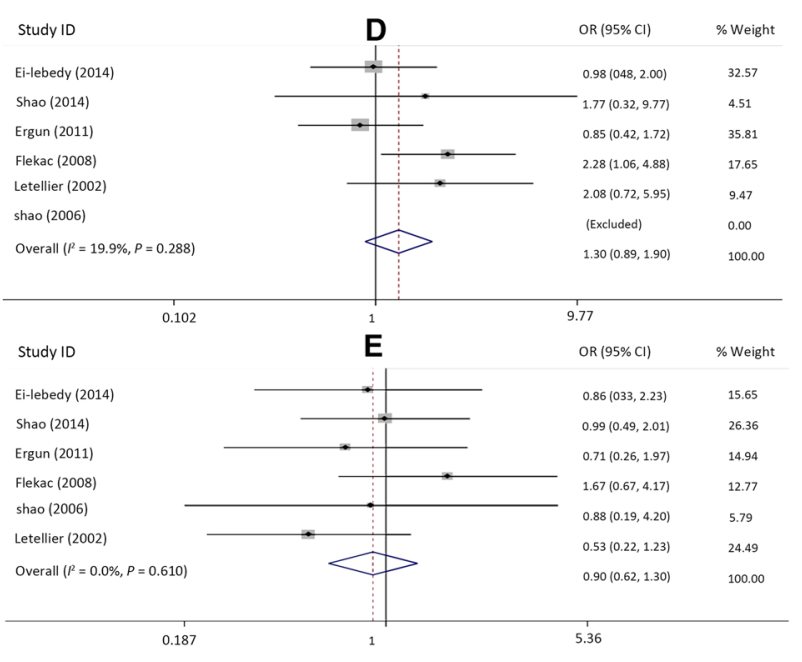

model, MM vs LL; c allelic contrast model, $\mathrm{M}$ vs $\mathrm{L}$; d recessive model, $\mathrm{MM}$ vs $\mathrm{LL}+\mathrm{LM}$; e heterozygous model, LM vs LL

significant publication bias in the models $(\mathrm{LM}+\mathrm{MM}$ vs LL: $t=-0.640, P=0.543 ; \mathrm{MM}$ vs LL: $t=-1.750, P=0.124 ; \mathrm{M}$ vs $\mathrm{L}: t=-0.710$, $P=0.503 ;$ LM vs LL: $t=-0.540, P=0.607$; data not shown) except for the recessive model (MM vs $L L+L M: t=2.580, \quad P=0.037$, data not shown).

\section{Paraoxonase 1 Q192R Genetic Polymorphism and Susceptibility to Diabetic Macroangiopathy}

As shown in Table 3, twelve studies focused on the association of paraoxonase 1 Q192R genetic polymorphism with the risk of diabetic macroangiopathy; these studies included 827 cases and 1069 controls [9, 10, 16, 17, 22, 23, 25-29, 32]. The results showed that there was significant heterogeneity in the dominant model $\left(\mathrm{QR}+\mathrm{RR}\right.$ vs QQ: $I^{2}=63.6 \%, P=0.001$, Fig. 3a, Table 4), homozygous model (RR vs QQ: $I^{2}=64.5 \%, P=0.001$, Fig. 3b, Table 4), allelic contrast model (R vs Q: $I^{2}=66.5 \%, P=0.001$, 

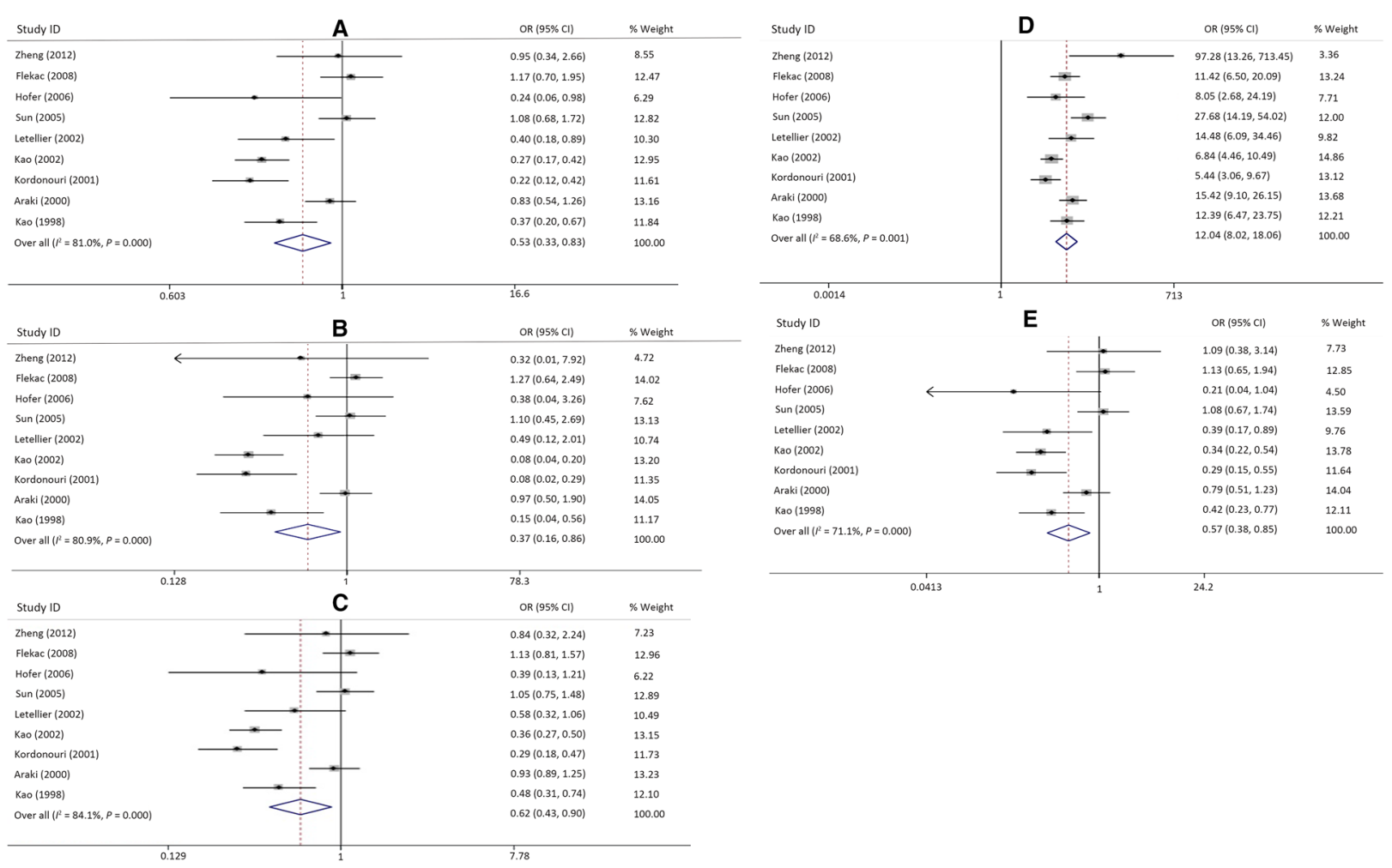

Fig. 2a-e Forest plots for the association of paraoxonase 1 L55M genetic polymorphism with diabetic microangiopathy. a Dominant model, LM + MM vs LL; b homozygous

Fig. 3c, Table 4), recessive model (RR vs QQ + QR: $I^{2}=45.7 \%, P=0.042$, Fig. 3d, Table 4), and heterozygous model (QR vs QQ: $I^{2}=52.5 \%$, $P=0.017$, Fig. 3e, Table 4). Subgroup analysis of ethnicity, type of DM, and HWE was therefore conducted, but it failed to find the sources of heterogeneity (Table 4). Meta-regression analysis was then performed using the covariates published year, sample size, ethnicity, type of DM, and HWE. The results showed that sample size could explain the heterogeneity in the dominant allelic and heterozygous models, and HWE could explain the heterogeneity in the homozygous and recessive models (Table 5). Paraoxonase 1 Q192R genetic polymorphism was significantly related to diabetic macroangiopathy susceptibility in the homozygous model (OR 1.88, 95\% CI 1.06-3.32, $P=0.030$, Fig. 3b, Table 4), allelic contrast model (OR 1.31 , 95\% CI $1.02-1.69, P=0.038$, Fig. 3c, Table 4), and recessive model (OR 1.55, 95\% CI 1.11-2.16, $P=0.010$, Fig. $3 d$, Table 4 ), but not

model, MM vs LL; c allelic contrast model, $\mathrm{M}$ vs $\mathrm{L}$; d recessive model, MM vs $L L+L M$; e heterozygous model, LM vs LL

the dominant model (OR 1.35, 95\% CI 0.88-2.08, $P=0.163$, Fig. 3a, Table 4) or heterozygous model (OR 1.20, 95\% CI $0.81-1.78, P=0.370$, Fig. 3e, Table 4). Sensitivity analysis indicated that when the effects models were changed, the statistical significance did not alter (data not shown). Meanwhile, there was no significant publication bias according to the funnel plot and Egger's test (QR + RR vs QQ: $t=1.44, P=0.179$; RR vs QQ: $t=1.46, P=0.174 ; \mathrm{R}$ vs $\mathrm{Q}: t=1.00, P=0.343$; RR vs QQ + QR: $t=1.30, P=0.224$; QR vs QQ: $t=1.03, P=0.326$; data not shown).

\section{Paraoxonase 1 Q192R Genetic Polymorphism and Susceptibility to Diabetic Microangiopathy}

As shown in Table 3, twelve studies focused on the association of paraoxonase 1 Q192R genetic polymorphism with the risk of diabetic 


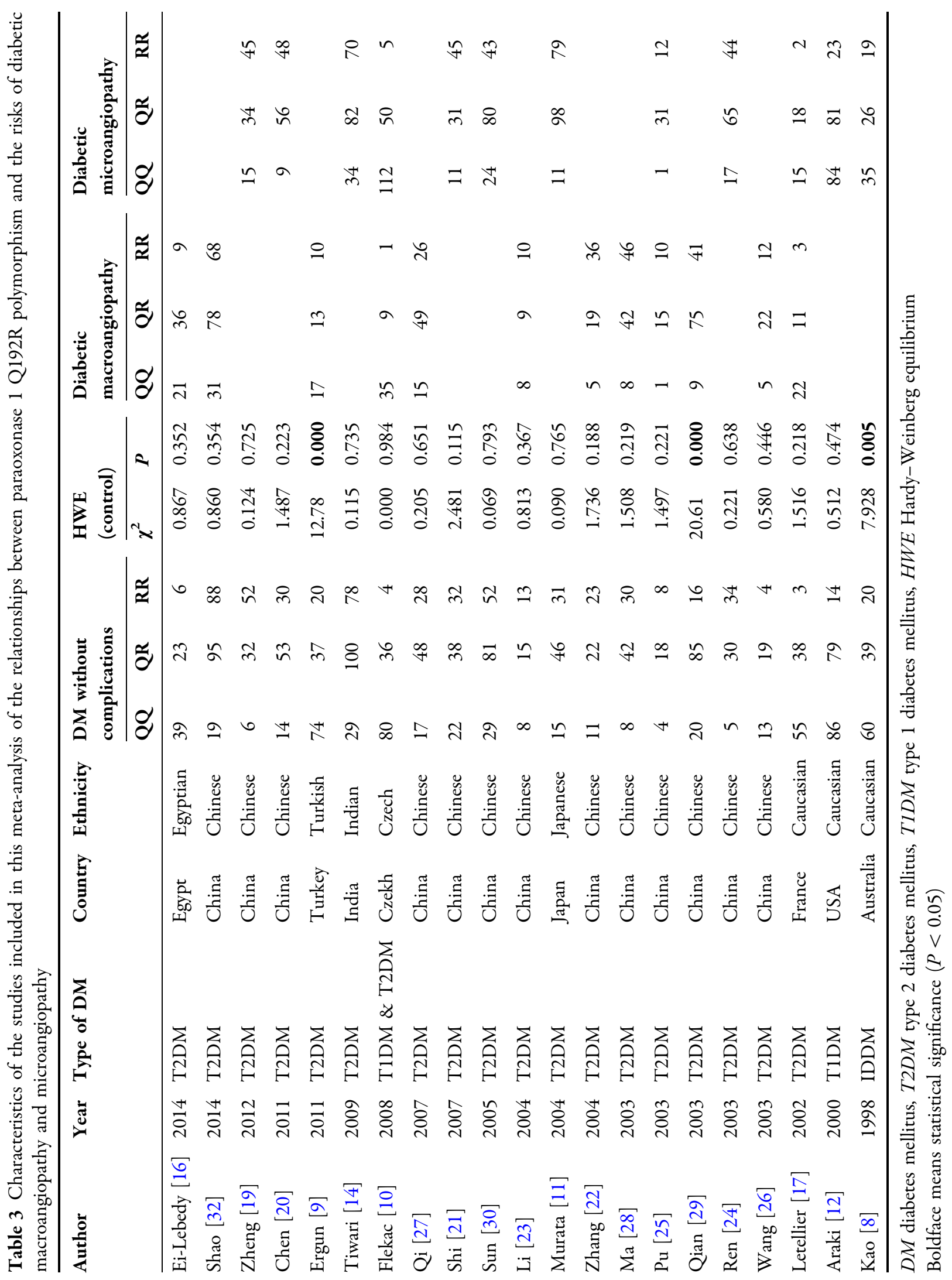




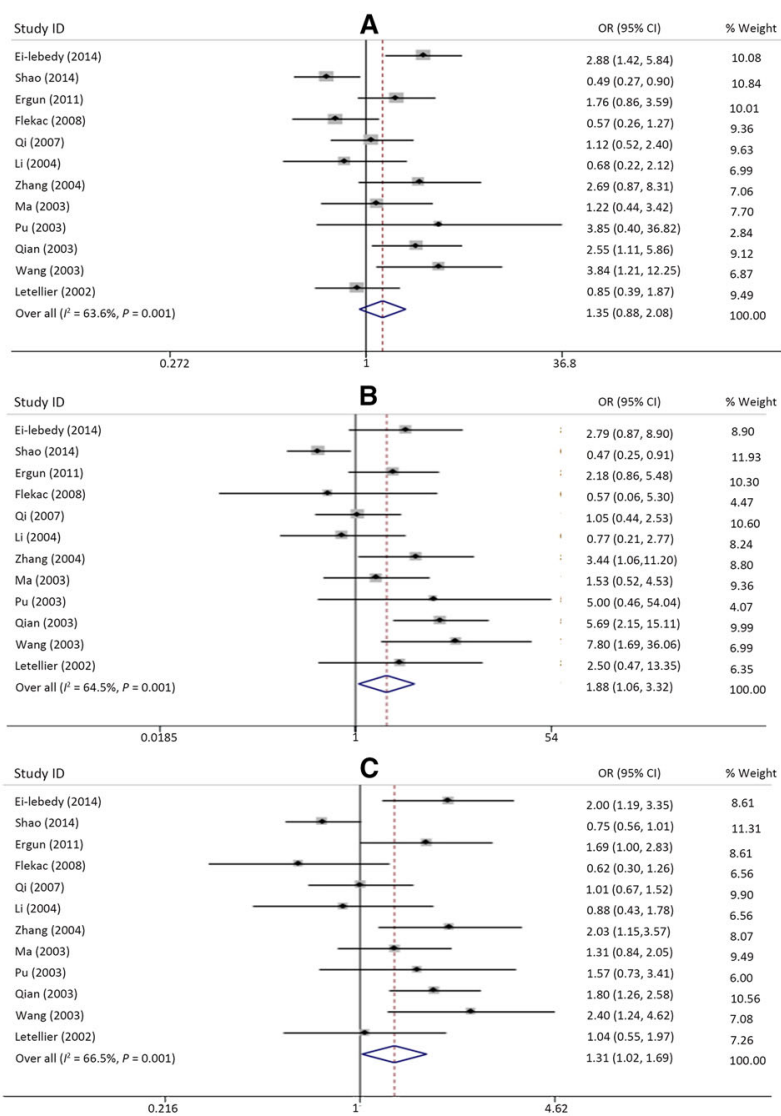

Fig. 3a-e Forest plots for the association of paraoxonase 1 Q192R genetic polymorphism with diabetic macroangiopathy. a Dominant model, $\mathrm{QR}+\mathrm{RR}$ vs $\mathrm{QQ}$;

microangiopathy; these studies included 1455 cases and 1353 controls $[8,10-12,14,17,19-21,24,25,30]$. The results showed that there was significant heterogeneity in the dominant model $(\mathrm{QR}+\mathrm{RR}$ vs QQ: $I^{2}=53.5 \%, \quad P=0.014, \quad$ Fig. $4 \mathrm{a}, \quad$ Table 4$)$, homozygote model (RR vs QQ: $I^{2}=60.4 \%$, $P=0.004$, Fig. 4 b, Table 4$)$, and allelic contrast model (R vs Q: $I^{2}=59.3 \%, P=0.005$, Fig. $4 \mathrm{c}$, Table 4) but not in the recessive model (RR vs QQ + QR: $\quad I^{2}=39.8 \%, \quad P=0.075, \quad$ Fig. $4 \mathrm{~d}$, Table 4) and heterozygous model (QR vs QQ: $I^{2}=35.1 \%, \quad P=0.109$, Fig. 4e, Table 4). Subgroup analysis of ethnicity, type of DM, and HWE and meta-regression analysis using the covariates published year, sample size, ethnicity, type of DM, and HWE were therefore performed to investigate the sources of heterogeneity. Unfortunately, the sources of

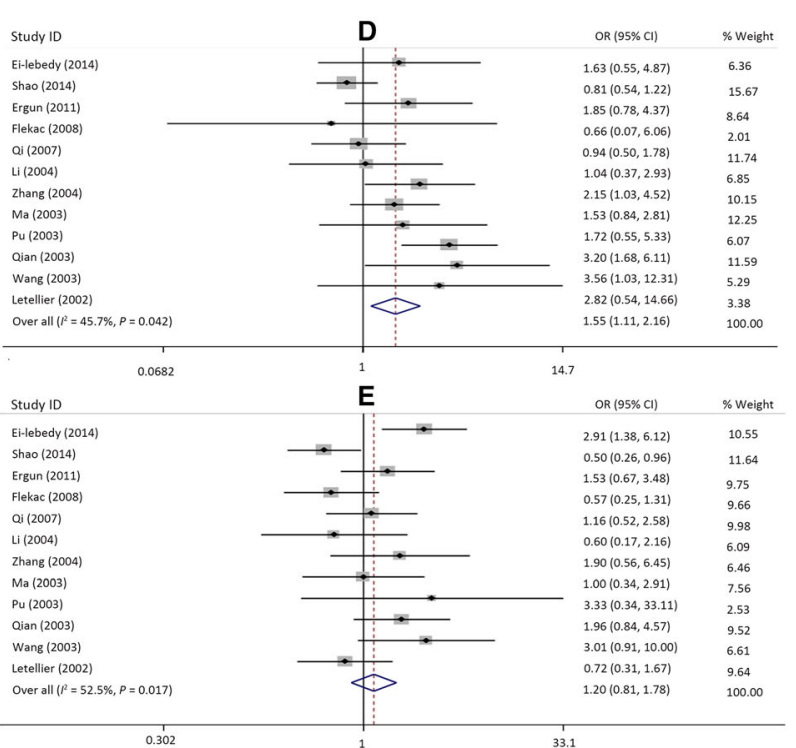

b homozygous model, RR vs QQ; c allelic contrast model, $\mathrm{R}$ vs $\mathrm{Q}$; $\mathbf{d}$ recessive model, $\mathrm{RR}$ vs $\mathrm{QQ}+\mathrm{QR}$; e heterozygous model, $\mathrm{QR}$ vs $\mathrm{QQ}$

heterogeneity were not found (Tables 4, 5). There was no marked association between paraoxonase 1 Q192R genetic polymorphism and susceptibility to diabetic microangiopathy in the dominant model (OR 1.21, 95\% CI 0.90-1.64, $P=0.209$, Fig. $4 a$, Table 4$)$, the homozygous model (OR 1.33, 95\% CI $0.86-2.05, P=0.119$, Fig. 4b, Table 4), the allelic contrast model (OR 1.12, 95\% CI 0.93-1.35, $P=0.227$, Fig. 4c, Table 4 ), the recessive model (OR 1.12, 95\% CI 0.93-1.33, $P=0.225$, Fig. 4d, Table 4), and the heterozygous model (OR 1.12, 95\% CI 0.92-1.36, $P=0.272$, Fig. 4e, Table 4). Sensitivity analysis indicated that when the effects models were changed, the statistical significances did not alter (data not shown). Meanwhile, according to the funnel plot and Egger's test, there was no significant publication bias in the models (QR + RR vs QQ: $t=2.19$, 
Table 4 Main results of the pooled data in this meta-analysis

\begin{tabular}{|c|c|c|c|c|c|c|c|c|c|c|c|c|c|c|c|}
\hline & \multicolumn{3}{|c|}{ Dominant model } & \multicolumn{3}{|c|}{ Homozygous model } & \multicolumn{3}{|c|}{ Allelic model } & \multicolumn{3}{|c|}{ Recessive model } & \multicolumn{3}{|c|}{ Heterozygous model } \\
\hline & OR $(95 \% \mathrm{CI})$ & $P$ & $\mathrm{I} 2, \%(P)$ & OR $(95 \% \mathrm{CI})$ & $P$ & $\mathrm{I} 2, \%(P)$ & OR $(95 \% \mathrm{CI})$ & $P$ & $\mathrm{I} 2, \%(P)$ & OR $(95 \% \mathrm{CI})$ & $P$ & $\mathrm{I} 2, \%(\mathrm{P})$ & $\mathrm{OR}(95 \% \mathrm{CI})$ & $P$ & $\mathrm{I} 2, \%(\mathrm{P})$ \\
\hline \multicolumn{16}{|c|}{ L55M DMMA } \\
\hline Total & $0.98(0.69-1.38)$ & 0.996 & $0.0(0.447)$ & $1.30(0.81-2.08)$ & 0.279 & $30.7(0.217)$ & $1.10(0.87-1.39)$ & 0.414 & $16.8(0.305)$ & $1.30(0.89-1.90)$ & 0.176 & $19.9(0.298)$ & $0.90(0.62-1.30)$ & 0.569 & $0.0(0.610)$ \\
\hline \multicolumn{16}{|c|}{ L55M DMMI } \\
\hline Total & $0.53(0.33-0.83)$ & 0.006 & $81.0(\mathbf{0 . 0 0 0})$ & $0.37(0.16-0.86)$ & 0.021 & $80.9(\mathbf{0 . 0 0 0 )}$ & $0.62(0.43-0.90)$ & 0.011 & $84.1(\mathbf{0 . 0 0 0})$ & $12.04(8.02-18.06)$ & 0.000 & $68.6(\mathbf{0 . 0 0 1})$ & $0.57(0.38-0.85)$ & 0.006 & $71.1(\mathbf{0 . 0 0 0})$ \\
\hline Asian & $1.06(0.69-1.62)$ & 0.794 & $0.0(0.827)$ & $1.01(0.43-2.38)$ & 0.988 & $0.0(0.465)$ & $1.03(0.74-1.42)$ & 0.871 & $0.0(0.676)$ & $38.41(11.68-126.37)$ & 0.000 & $40.0(0.197)$ & $1.08(0.70-1.67)$ & 0.729 & $0.0(0.986)$ \\
\hline NAS & $0.44(0.26-0.74)$ & 0.002 & $81.1(\mathbf{0 . 0 0 0})$ & $0.31(0.12-0.84)$ & 0.022 & $84.4(\mathbf{0 . 0 0 0})$ & $0.55(0.36-0.84)$ & 0.006 & $85.7(\mathbf{0 . 0 0 0})$ & $9.73(7.06-13.41)$ & 0.000 & $46.2(0.084)$ & $0.48(0.31-0.74)$ & 0.001 & $69.8(\mathbf{0 . 0 0 3})$ \\
\hline T2DM & $0.77(0.41-1.46)$ & 0.424 & $55.8(0.104)$ & $0.83(0.40-1.73)$ & 0.614 & $0.0(0.531)$ & $0.86(0.58-1.26)$ & 0.435 & $29.8(0.241)$ & $25.4(11.28-57.48)$ & 0.000 & $49.0(0.141)$ & $0.79(0.40-1.54)$ & 0.483 & $56.7(0.099)$ \\
\hline Other & $0.44(0.25-0.80)$ & 0.006 & $84.1(\mathbf{0 . 0 0 0})$ & $0.29(0.09-0.89)$ & 0.031 & $87.0(\mathbf{0 . 0 0 0 )}$ & $0.54(0.34-0.88)$ & 0.014 & $88.1(\mathbf{0 . 0 0 0})$ & $9.33(6.60-13.19)$ & 0.000 & $50.8(0.071)$ & $0.49(0.30-0.80)$ & 0.004 & $74.1(\mathbf{0 . 0 0 2})$ \\
\hline HWE & $0.57(0.33-1.01)$ & 0.053 & $84.5(\mathbf{0 . 0 0 0})$ & $0.40(0.15-1.10)$ & 0.076 & $84.3(\mathbf{0 . 0 0 0})$ & $0.95(0.41-1.02)$ & 0.061 & $87.4(\mathbf{0 . 0 0 0})$ & $11.92(7.12-19.96)$ & 0.000 & $75.9(\mathbf{0 . 0 0 0})$ & $0.62(0.39-1.01)$ & 0.055 & $76.2(\mathbf{0 . 0 0 0})$ \\
\hline NH & $0.38(0.33-0.83)$ & 0.000 & $0.0(0.862)$ & $0.26(0.08-0.86)$ & 0.024 & $30.8(0.229)$ & $0.51(0.43-0.90)$ & 0.000 & $0.0(0.620)$ & $13.11(7.79-22.06)$ & 0.000 & $0.0(0.778)$ & $0.41(0.25-0.66)$ & 0.000 & $0.0(0.882)$ \\
\hline \multicolumn{16}{|c|}{ Q192R DMMA } \\
\hline Total & $1.35(0.88-2.08)$ & 0.163 & $63.6(0.001)$ & $1.88(1.06,3.32)$ & 0.030 & $64.5(0.001)$ & $1.31(1.02,1.69)$ & 0.038 & $66.5(0.001)$ & $1.55(1.11-2.16)$ & 0.010 & $45.7(\mathbf{0 . 0 4 2})$ & $1.20(0.81-1.78)$ & 0.370 & $52.5(0.017)$ \\
\hline Asian & $1.58(0.98-2.56)$ & 0.061 & $63.5(\mathbf{0 . 0 0 3})$ & $1.97(1.05-3.69)$ & 0.034 & $69.9(\mathbf{0 . 0 0 0 )}$ & $1.41(1.07-1.86)$ & 0.013 & $68.5(0.001)$ & $1.55(1.09-2.21)$ & 0.014 & $52.9(\mathbf{0 . 0 2 4})$ & $1.39(0.90-2.16)$ & 0.142 & $51.3(\mathbf{0 . 0 3 0})$ \\
\hline NAS & $0.70(0.40-1.23)$ & 0.213 & $0.0(0.482)$ & $1.44(0.35-5.89)$ & 0.611 & $8.4(0.296)$ & $0.82(0.50-1.36)$ & 0.448 & $11.0(28.9)$ & $1.66(0.42-6.59)$ & 0.474 & $7.0(0.300)$ & $0.64(0.36-1.16)$ & 0.141 & $0.0(0.694)$ \\
\hline T2DM & $1.48(0.95-2.29)$ & 0.082 & $61.8(\mathbf{0 . 0 0 3})$ & $1.99(1.10-3.59)$ & 0.022 & $66.9(0.001)$ & $1.38(1.07-1.78)$ & 0.014 & $65.5(\mathbf{0 . 0 0 1 )}$ & $1.58(1.13-2.23)$ & 0.008 & $49.6(\mathbf{0 . 0 3 1})$ & $1.30(0.86-1.95)$ & 0.216 & $50.5(\mathbf{0 . 0 2 7})$ \\
\hline Other & NA & NA & NA (NA) & $\mathrm{NA}$ & NA & NA (NA) & NA & NA & NA (NA) & NA & NA & NA (NA) & NA & NA & NA (NA) \\
\hline HWE & $1.23(0.75-2.01)$ & 0.414 & $65.0(\mathbf{0 . 0 0 2})$ & $1.59(0.86-2.93)$ & 0.138 & $58.9(\mathbf{0 . 0 0 9})$ & $1.22(0.92-1.63)$ & 0.167 & $65.2(\mathbf{0 . 0 0 2})$ & $1.31(0.96-1.80)$ & 0.088 & $25.8(0.206)$ & $1.11(0.69-1.76)$ & 0.672 & $56.3(\mathbf{0 . 0 1 4})$ \\
\hline NH & $2.06(1.20-3.54)$ & 0.009 & $0.0(0.504)$ & $3.47(1.35-3.32)$ & 0.010 & $49.2(0.161)$ & $1.76(1.31-2.37)$ & 0.000 & $0.0(0.835)$ & $2.63(1.57-4.41)$ & 0.000 & $0.6(0.316)$ & $1.73(0.8-1.78)$ & 0.070 & $0.0(0.680)$ \\
\hline \multicolumn{16}{|c|}{ Q192R DMMI } \\
\hline Total & $1.21(0.90-1.64)$ & 0.209 & $53.5(\mathbf{0 . 0 1 4})$ & $1.33(0.86-2.05)$ & 0.119 & $60.4(\mathbf{0 . 0 0 4})$ & $1.12(0.93-1.35)$ & 0.227 & $59.3(\mathbf{0 . 0 0 5})$ & $1.12(0.93-1.33)$ & 0.225 & $39.8(0.075)$ & $1.12(0.92-1.36)$ & 0.272 & $35.1(0.109)$ \\
\hline Asian & $1.22(0.72-2.08)$ & 0.456 & $68.0(\mathbf{0 . 0 0 3})$ & $1.26(0.68-2.34)$ & 0.468 & $72.9(\mathbf{0 . 0 0 1})$ & $1.07(0.83-1.40)$ & 0.595 & $71.0(\mathbf{0 . 0 0 1})$ & $1.06(0.87-1.28)$ & 0.579 & $55.0(\mathbf{0 . 0 3 0})$ & $1.12(0.84-1.50)$ & 0.430 & $54.8(\mathbf{0 . 0 3 0})$ \\
\hline NAS & $1.19(0.91-1.54)$ & 0.2 & $0.0(0.626)$ & $1.57(0.98-2.52)$ & 0.06 & $0.0(0.818)$ & $1.21(0.99-1.48)$ & 0.067 & $0.0(0.611)$ & $1.50(0.96-2.36)$ & 0.076 & $0.0(0.873)$ & $1.11(0.85-1.47)$ & 0.444 & $0.0(0.692)$ \\
\hline T2DM & $1.28(0.80-2.05)$ & 0.311 & $65.2(\mathbf{0 . 0 0 3})$ & $1.31(0.73-2.36)$ & 0.368 & $69.7(\mathbf{0 . 0 0 1})$ & $1.11(0.86-1.42)$ & 0.422 & $68.7(\mathbf{0 . 0 0 1})$ & $1.06(0.88-1.29)$ & 0.537 & $49.7(\mathbf{0 . 0 4 4})$ & $1.18(0.90-1.55)$ & 0.230 & $51.5(\mathbf{0 . 0 3 6})$ \\
\hline Other & $1.31(0.85-1.49)$ & 0.398 & $0.0(0.756)$ & $1.53(0.94-2.49)$ & 0.09 & $0.0(0.702)$ & $1.17(0.94-1.45)$ & 0.149 & $0.0(0.570)$ & $1.49(0.93-2.36)$ & 0.095 & $0.0(0.725)$ & $1.05(0.78-1.41)$ & 0.752 & $0.0(0.945)$ \\
\hline HWE & $1.21(0.86-1.69)$ & 0.274 & $57.5(\mathbf{0 . 0 0 9})$ & $1.30(0.80-2.11)$ & 0.284 & $63.4(\mathbf{0 . 0 0 2})$ & $1.10(0.90-1.34)$ & 0.336 & $61.7(\mathbf{0 . 0 0 4 )}$ & $1.09(0.91-1.31)$ & 0.346 & $42.5(0.066)$ & $1.12(0.90-1.38)$ & 0.306 & $41.0(0.075)$ \\
\hline $\mathrm{NH}$ & NA & NA & NA (NA) & NA & NA & $\mathrm{NA}(\mathrm{NA})$ & NA & NA & $\mathrm{NA}(\mathrm{NA})$ & NA & NA & $\mathrm{NA}(\mathrm{NA})$ & NA & NA & $\mathrm{NA}(\mathrm{NA})$ \\
\hline
\end{tabular}

$D M M A$ diabetic macroangiopathy, DMMI diabetic microangiopathy, T2DM type 2 diabetes mellitus, NAS non-Asian, $N A$ not available, $H W E$ Hardy-Weinberg equilibrium, $N H$ non-HWE

$P=0.054 ; \mathrm{RR}$ vs QQ: $t=1.35, P=0.207$; R vs $\mathrm{Q}:$ $t=0.69, P=0.505$, RR vs QQ $+\mathrm{QR}: t=0.61$, $P=0.555$; data not shown) except in the heterozygous model (QR vs QQ: $t=2.36$, $P=0.040$, data not shown).

\section{DISCUSSION}

This study is the first to demonstrate that paraoxonase-1 L55M and Q192R genetic polymorphisms are associated with susceptibility to diabetic macroangiopathy and microangiopathy. The results showed that paraoxonase 1 L55M genetic polymorphism was significantly related to diabetic microangiopathy susceptibility, but not diabetic macroangiopathy susceptibility, in the dominant, homozygous, and allelic contrast models. There was also a significant association of paraoxonase 1 Q192R genetic polymorphism with diabetic macroangiopathy susceptibility in the homozygous and allelic contrast models, but not in the dominant model. Meanwhile, there was no significant relationship between paraoxonase 1 Q192R genetic polymorphism and susceptibility to diabetic microangiopathy.

Paraoxonase 1 has been shown to have antioxidant properties and to protect low- 


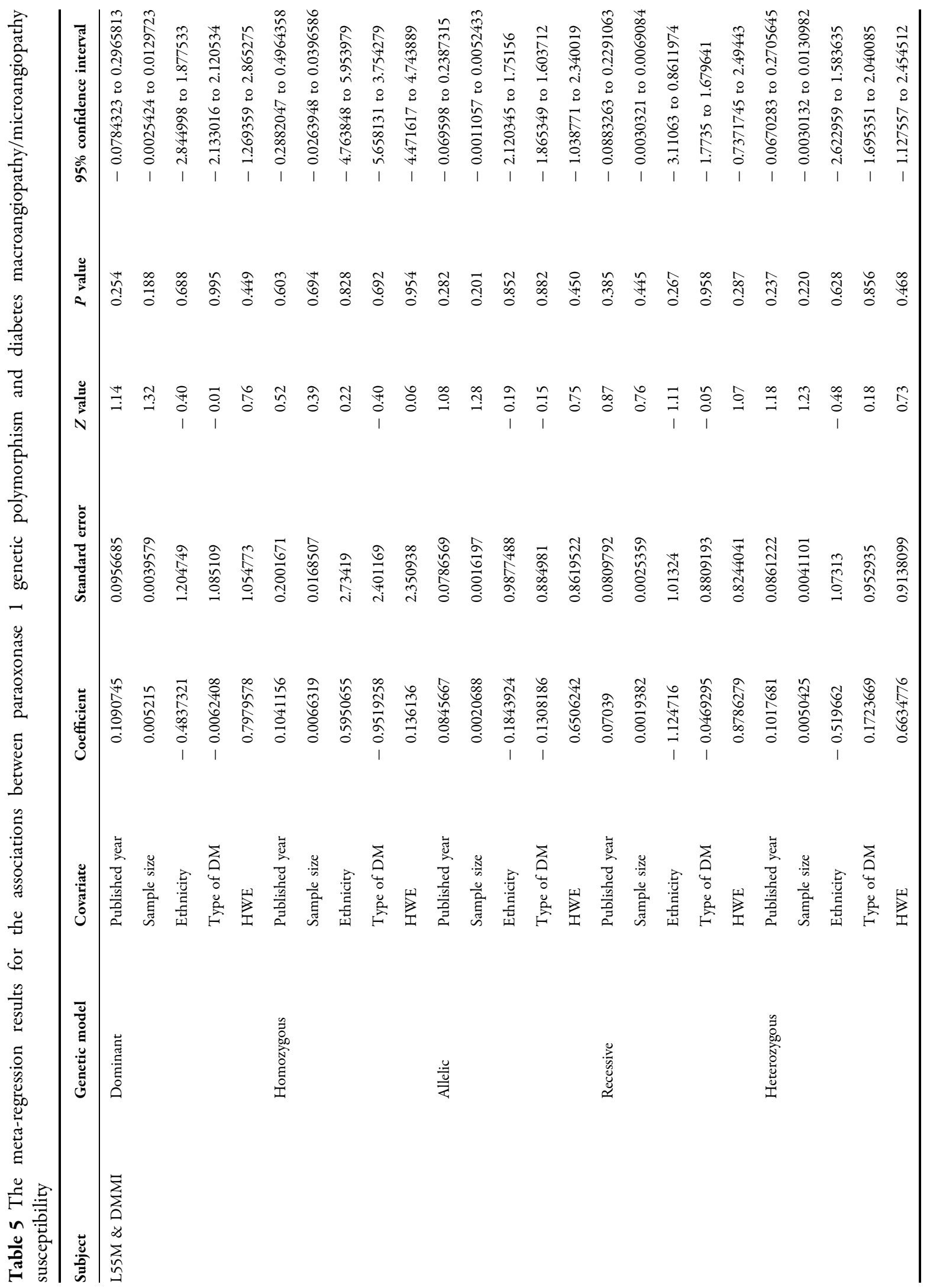




\begin{tabular}{|c|c|c|c|c|c|c|c|c|c|c|c|c|c|c|c|c|c|c|c|c|c|c|c|}
\hline 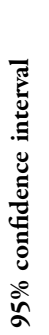 & 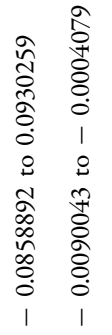 & 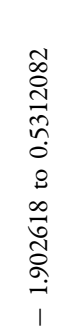 & 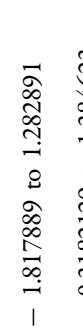 & 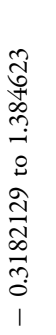 & 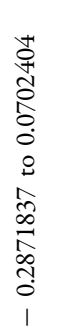 & \begin{tabular}{l}
$\hat{a}$ \\
$\hat{0}$ \\
0 \\
0 \\
0 \\
0 \\
0 \\
\multirow{0}{*}{} \\
$\frac{1}{7}$ \\
0 \\
0 \\
0 \\
0 \\
1
\end{tabular} & 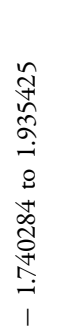 & 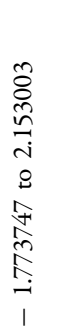 & 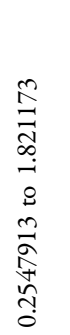 & \begin{tabular}{l}
0 \\
1 \\
0 \\
0 \\
0 \\
0 \\
0 \\
0 \\
0 \\
0 \\
\multirow{3}{*}{} \\
0 \\
0 \\
0 \\
1
\end{tabular} & 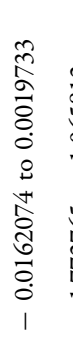 & 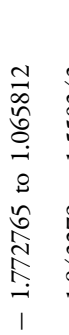 & 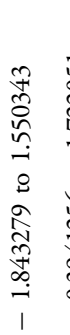 & 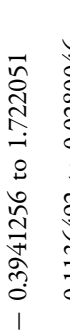 & 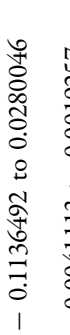 & 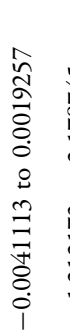 & 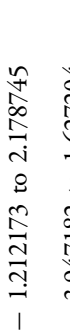 & 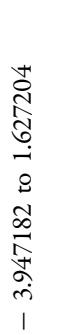 & 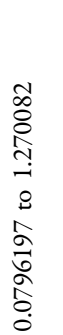 & 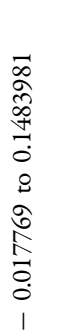 & 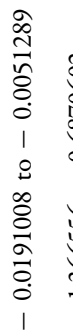 & & 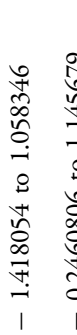 \\
\hline 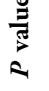 & @̂̃ & त्ठ & 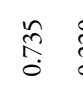 & तี & त्ञ & 命 & $\stackrel{\widehat{a}}{\circ}$ & 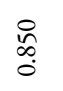 & $\stackrel{\text { ڤे }}{0}$ & 苦 & $\stackrel{n}{\approx}$ & $\begin{array}{l}\text { त̂े } \\
\text { ठ } \\
0\end{array}$ & $\begin{array}{l}0 \\
0 \\
\infty \\
0\end{array}$ & خे & 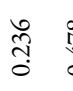 & \begin{tabular}{l}
$\infty$ \\
$\stackrel{c}{+}$ \\
\multirow{0}{*}{}
\end{tabular} & है & $\underset{f}{n}$ & 苞 & $\stackrel{\widetilde{3}}{0}$ & $\ddot{\Xi}$ & $\hat{\tilde{n}}$ & $\begin{array}{l}0 \\
\hat{o} \\
\text { ô }\end{array}$ \\
\hline 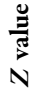 & $\begin{array}{ccc}\infty & n \\
0 & \stackrel{n}{i} & \\
& 1\end{array}$ & $\stackrel{\bigodot}{\rightarrow}$ & $\begin{array}{l}\text { 苦 } \\
0 \\
1\end{array}$ & 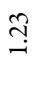 & $\underset{\vec{t}}{\vec{t}}$ & $\begin{array}{l}\text { के } \\
\text { i } \\
1\end{array}$ & $\stackrel{0}{\circ}$ & $\stackrel{2}{0}$ & i & $\begin{array}{l}\text { t? } \\
0\end{array}$ & $\stackrel{\tilde{n}}{\stackrel{i}{i}}$ & $\begin{array}{l}\text { Fे } \\
0 \\
1\end{array}$ & $\begin{array}{l}\hat{0} \\
i\end{array}$ & $\stackrel{\overbrace{}}{\rightarrow}$ & $\stackrel{\overbrace{}}{\leftrightarrows}$ & $\begin{array}{l}\text { त् } \\
\text { i } \\
i\end{array}$ & $\stackrel{\wp}{0}$ & $\begin{array}{l}0 \\
\infty \\
0 \\
1 \\
1\end{array}$ & $\begin{array}{l}\tilde{N} \\
\text {. }\end{array}$ & 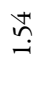 & 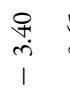 & $\begin{array}{l}\tilde{n} \\
0 \\
i\end{array}$ & 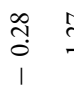 \\
\hline 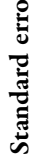 & 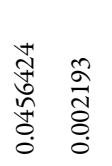 & $\begin{array}{l}\hat{\hat{\alpha}} \\
0 \\
\hat{\sigma} \\
0 \\
0\end{array}$ & 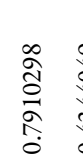 & 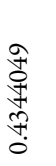 & $\begin{array}{l}\stackrel{\infty}{\infty} \\
\stackrel{\infty}{*} \\
0 \\
0\end{array}$ & $\begin{array}{l}\infty \\
\infty \\
o \\
0 \\
0 \\
0\end{array}$ & $\begin{array}{l}\vec{\infty} \\
\delta \\
0 \\
\hat{\alpha} \\
0\end{array}$ & $\begin{array}{l}\stackrel{+}{c} \\
\stackrel{5}{\circ}\end{array}$ & 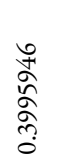 & $\begin{array}{l}a \\
\hat{a} \\
\hat{\sigma} \\
0 \\
0 \\
0\end{array}$ & 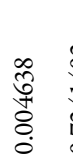 & 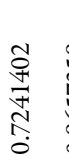 & $\begin{array}{l}\infty \\
\hat{n} \\
\hat{\hat{\alpha}} \\
\infty \\
0 \\
0\end{array}$ & 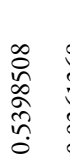 & $\begin{array}{l}\infty \\
0 \\
0 \\
0 \\
0 \\
0 \\
0\end{array}$ & $\begin{array}{l}\overrightarrow{0} \\
\stackrel{+}{1} \\
\stackrel{0}{0} \\
0 \\
0\end{array}$ & 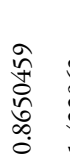 & 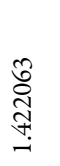 & 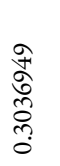 & 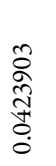 & 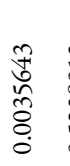 & & 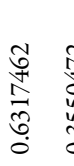 \\
\hline 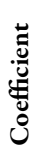 & 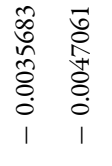 & $\begin{array}{l}n \\
\hat{Q} \\
\hat{人} \\
00 \\
0 \\
1 \\
1\end{array}$ & 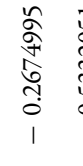 & $\begin{array}{l}\bar{n} \\
\text { त̂̀ } \\
\hat{n} \\
\hat{o}\end{array}$ & $\begin{array}{l}\text { 章 } \\
\text { o } \\
0 \\
0 \\
0 \\
1\end{array}$ & 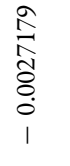 & $\begin{array}{l}\hat{n} \\
\hat{o} \\
0\end{array}$ & 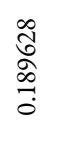 & 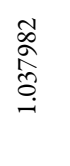 & $\begin{array}{l}\tilde{N} \\
\hat{\tilde{N}} \\
\hat{O} \\
0\end{array}$ & 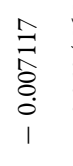 & 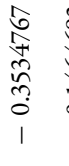 & $\begin{array}{l}0 \\
0 \\
0 \\
+0 \\
0 \\
+1 \\
0 \\
1 \\
1\end{array}$ & 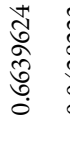 & \begin{tabular}{l} 
तิ \\
o \\
\multirow{3}{0}{} \\
$\dot{0}$ \\
1
\end{tabular} & 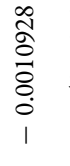 & 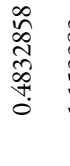 & $\begin{array}{l}\stackrel{a}{\alpha} \\
\hat{\sigma} \\
\stackrel{\vec{n}}{i} \\
1\end{array}$ & $\begin{array}{l}\infty \\
0 \\
\infty \\
\alpha+1 \\
0 \\
0 \\
0 \\
0\end{array}$ & 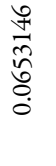 & $\begin{array}{l}\underset{\infty}{\mathbb{Z}} \\
\underset{\Xi}{\Xi} \\
\stackrel{0}{0} \\
1\end{array}$ & 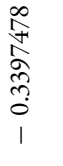 & 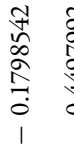 \\
\hline نे & 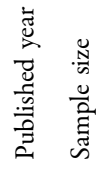 & 总 & 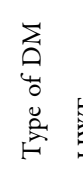 & I & 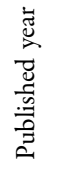 & 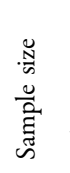 & 栉 & 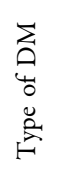 & 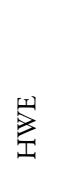 & 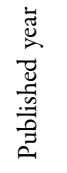 & 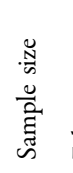 & 㺼 & 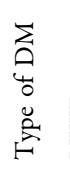 & : & 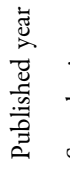 & 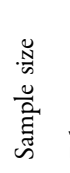 & 总 & 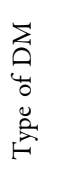 & 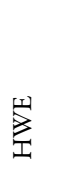 & 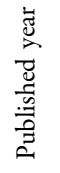 & 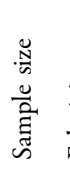 & 总 & 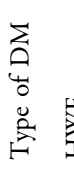 \\
\hline 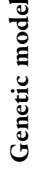 & 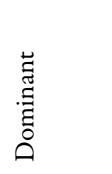 & & & & 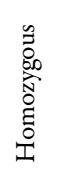 & & & & & 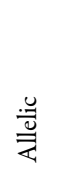 & & & & & 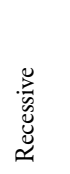 & & & & & 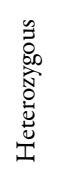 & & & \\
\hline 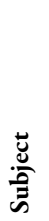 & 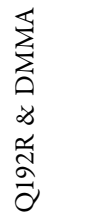 & & & & & & & & & & & & & & & & & & & & & & \\
\hline
\end{tabular}




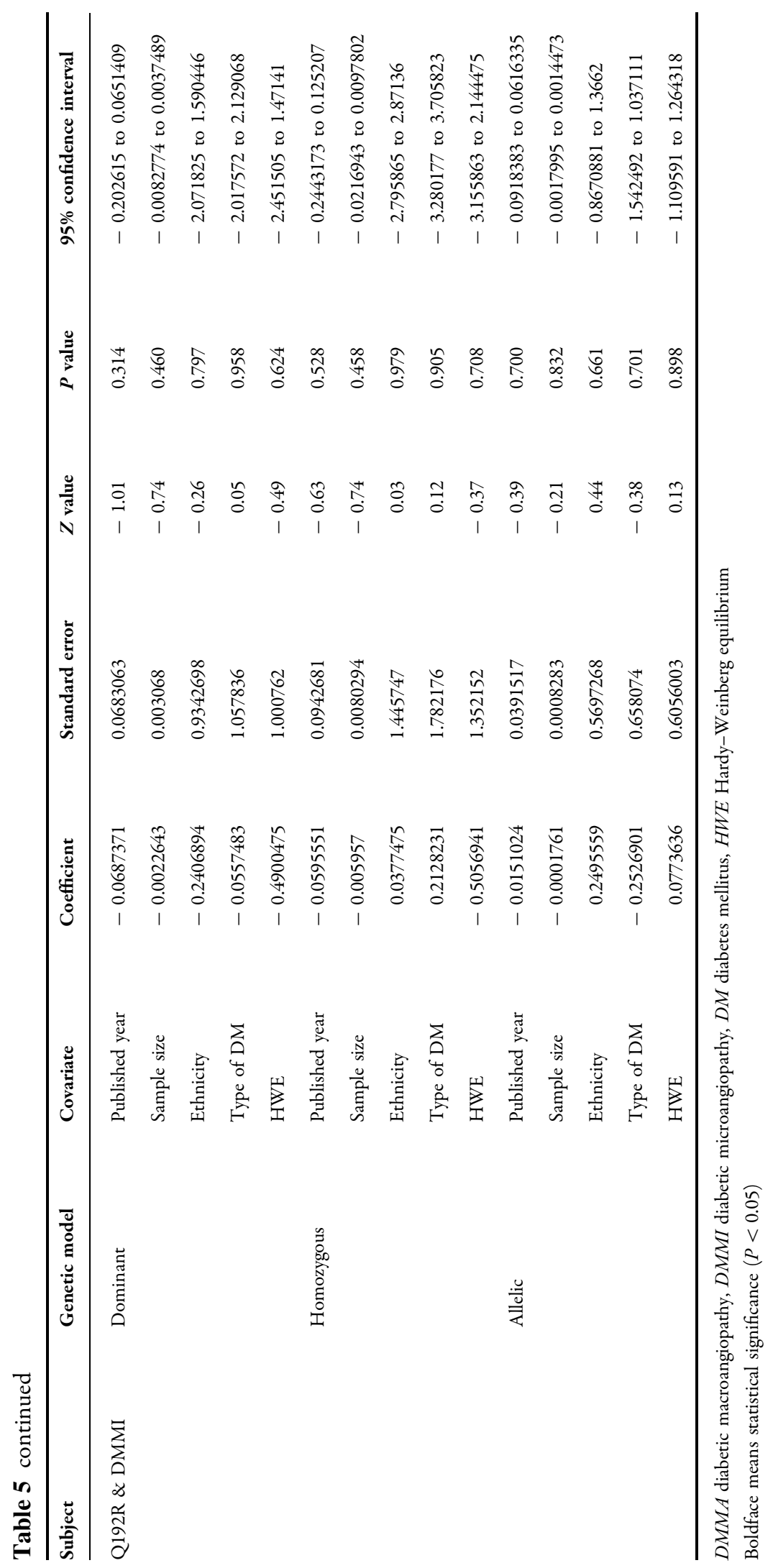




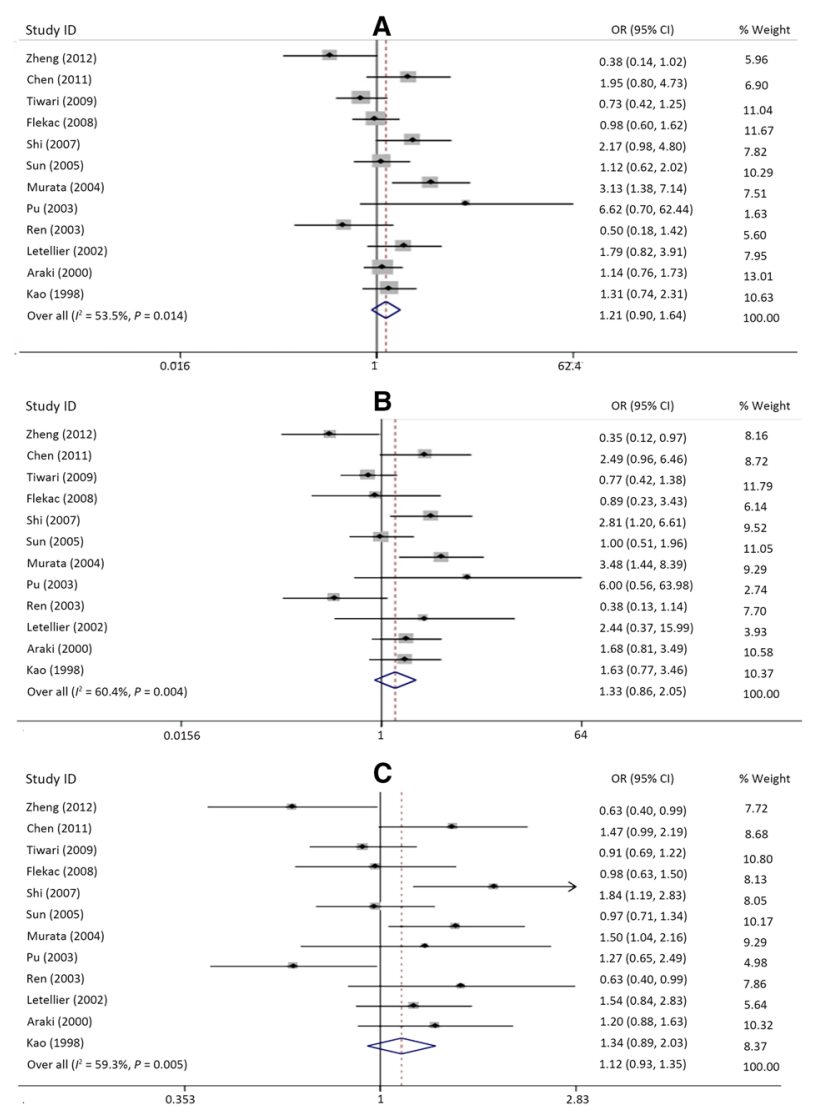

Fig. 4a-e Forest plots for the association of paraoxonase 1 Q192R genetic polymorphism with diabetic microangiopathy. a Dominant model, $\mathrm{QR}+\mathrm{RR}$ vs $\mathrm{QQ}$;

density lipoprotein from oxidative damage. The 192Q allele of paraoxonase 1 is more effective than $192 \mathrm{R}$ at preventing low-density lipoprotein oxidation [33]. The 192R allele was found to be significantly associated with lower serum paraoxonase 1 activity in DM patients in an Egyptian population [16]. A recent meta-analysis also demonstrated that Q192R genetic polymorphism was significantly related to the risk of type 2 diabetes mellitus, although there were some ethnic differences [7]. However, the relationships between paraoxonase 1 Q192R genetic polymorphism and various diabetic complications are considered to be rather unclear. Therefore, the meta-analysis reported in the present paper was performed to examine these relationships, and we found that there was a significant relationship between paraoxonase 1 Q192R genetic polymorphism and

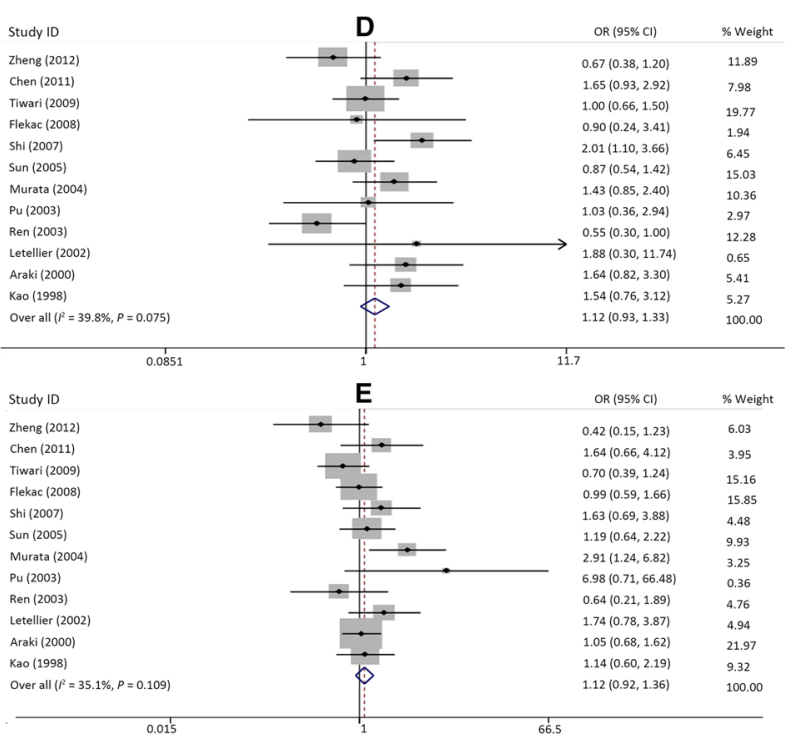

b homozygous model, RR vs QQ; c allelic contrast model, $\mathrm{R}$ vs $\mathrm{Q}$; $\mathbf{d}$ recessive model, $\mathrm{RR}$ vs $\mathrm{QQ}+\mathrm{QR}$; e heterozygous model, QR vs QQ

susceptibility to diabetic macroangiopathy in the homozygous and allelic contrast models, but not in the dominant model. Meanwhile, there was no significant association between paraoxonase 1 Q192R genetic polymorphism and diabetic microangiopathy susceptibility. However, there were significant heterogeneities in all three of these models when the associations of Q192R with diabetic macroangiopathy and microangiopathy susceptibility were analyzed. Meta-regression analysis was performed using the covariates published year, sample size, ethnicity, and type of DM, but it failed to identify the sources of heterogeneity.

Paraoxonase 1 L55M genetic polymorphism, another common single nucleotide polymorphism of paraoxonase 1 , can affect the enzyme activity of paraoxonase 1 , with the $\mathrm{L}$ allele linked to a higher serum concentration and 
greater stability to proteolysis [33, 34]. A recent meta-analysis found that L55M polymorphism was significantly associated with many diseases, such as any cancer, breast cancer [35], and diabetes mellitus [7]. Paraoxonase 1 L55M genetic polymorphism was also found to be significantly associated with diabetic retinopathy in a meta-analysis which included just two articles [36]. Thus, before the present meta-analysis was carried out, the relationships of L55M genetic polymorphism with diabetic macroangiopathy and microangiopathy were not very clear. In this meta-analysis, L55M genetic polymorphism was found to be significantly associated with susceptibility to diabetic microangiopathy but not susceptibility to diabetic macroangiopathy in the dominant, homozygous, and allelic contrast models. Thus, subgroup analysis and meta-regression were conducted, and the results showed that type of DM was able to explain the heterogeneity in the recessive model, but not in the other models.

It is important to note the limitations of this meta-analysis. First, only articles written in English and Chinese were included in this study, and there may have been some publication bias, although Egger's test and a funnel plot did not point to any significant publication bias. Second, we used composites of diabetic complications in the present meta-analysis. Although macroangiopathy and microangiopathy are two kinds of diabetic vascular complications, the pathophysiological mechanisms and the contributions of genetic polymorphisms were probably different for these complications. Third, significant heterogeneity was found in our analysis, but subgroup analysis and meta-regression failed to find any of the sources of the between-study heterogeneity in the associations of paraoxonase 1 L55M/Q192R genetic polymorphisms with the risk of diabetic microangiopathy. Therefore, further well-designed studies with large samples are needed to confirm the results of this meta-analysis.

\section{CONCLUSIONS}

Paraoxonase 1 L55M and Q192R genetic polymorphisms play important roles in the susceptibility to diabetic macroangiopathy and microangiopathy.

\section{ACKNOWLEDGEMENTS}

Funding. This research was supported by the National Natural Science Foundation of China (no. 81701962) and the Natural Science Foundation of Hunan (no. 2018JJ2589). The article processing charges were funded by the authors. All authors had full access to all of the data in this study and take complete responsibility for the integrity of the data and accuracy of the data analysis.

Authorship. All named authors meet the International Committee of Medical Journal Editors (ICMJE) criteria for authorship for this article, take responsibility for the integrity of the work as a whole, and have given their approval for this version to be published.

Disclosures. Chenfang $\mathrm{Wu}$, Diling $\mathrm{Wu}$, Minjie Lin, and Yanjun Zhong have nothing to disclose.

Compliance with Ethics Guidelines. This article is based on previously conducted studies and does not contain any studies with human participants or animals performed by any of the authors.

Data Availability. The datasets obtained during and/or analyzed during the current study are available from the corresponding author on reasonable request.

Open Access. This article is distributed under the terms of the Creative Commons Attribution-NonCommercial 4.0 International License (http://creativecommons.org/licenses/ by-nc/4.0/), which permits any noncommercial use, distribution, and reproduction in any medium, provided you give appropriate credit to the original author(s) and the source, provide a link to the Creative Commons license, and indicate if changes were made. 


\section{REFERENCES}

1. Madonna R, Pieragostino D, Balistreri CR, et al. Diabetic macroangiopathy: pathogenetic insights and novel therapeutic approaches with focus on high glucose-mediated vascular damage. Vasc Pharmacol. 2018;S1537-1891:30322-1.

2. Barrett EJ, Liu Z, Khamaisi M, et al. Diabetic microvascular disease: an Endocrine Society scientific statement. J Clin Endocrinol Metab. 2017;102(12):4343-410. https://doi.org/10.1210/jc.2017-01922.

3. Vakili L, Navab KD, Shabihkhani M, et al. Systemic inflammation, intestine, and paraoxonase-1. Adv Exp Med Biol. 2014;824:83-8.

4. Poh R, Muniandy S. Paraoxonase 1 activity as a predictor of cardiovascular disease in type 2 diabetes. Southeast Asian J Trop Med Public Health. 2010;41:1231-46.

5. Mackness B, Durrington PN, Abuashia B, Boulton AJ, Mackness MI. Low paraoxonase activity in type II diabetes mellitus complicated by retinopathy. Clin Sci (Lond). 2000;98:355-63.

6. Abdin AA, Hassanien MA, Ibrahim EA, El-Noeman SD. Modulating effect of atorvastatin on paraoxonase 1 activity in type 2 diabetic Egyptian patients with or without nephropathy. J Diabetes Complicat. 2010;24:325-33.

7. Luo JQ, Ren H, Liu MZ, Fang PF, Xiang DX. European versus Asian differences for the associations between paraoxonase-1 genetic polymorphisms and susceptibility to type 2 diabetes mellitus. J Cell Mol Med. 2018;22:1720-32.

8. Kao YL, Donaghue K, Chan A, Knight J, Silink M. A variant of paraoxonase (PON1) gene is associated with diabetic retinopathy in IDDM. J Clin Endocrinol Metab. 1998;83:2589-92.

9. Ergun MA, Yurtcu E, Demirci H, et al. PON1 55 and 192 gene polymorphisms in type 2 diabetes mellitus patients in a Turkish population. Biochem Genet. 2011;49:1-8.

10. Flekac M, Skrha J, Zidkova K, Lacinova Z, Hilgertova J. Paraoxonase 1 gene polymorphisms and enzyme activities in diabetes mellitus. Physiol Res. 2008;57:717-26.

11. Murata M, Maruyama T, Suzuki Y, Saruta T, Ikeda Y. Paraoxonase $1 \mathrm{Gln} /$ Arg polymorphism is associated with the risk of microangiopathy in type 2 diabetes mellitus. Diabet Med. 2004;21:837-44.

12. Araki S, Makita Y, Canani L, Ng D, Warram JH, Krolewski AS. Polymorphisms of human paraoxonase 1 gene (PON1) and susceptibility to diabetic nephropathy in type I diabetes mellitus. Diabetologia. 2000;43:1540-3.

13. Kao Y, Donaghue KC, Chan A, Bennetts BH, Knight J, Silink M. Paraoxonase gene cluster is a genetic marker for early microvascular complications in type 1 diabetes. Diabet Med. 2002;19:212-5.

14. Tiwari AK, Prasad P, Thelma BK, et al. Oxidative stress pathway genes and chronic renal insufficiency in Asian Indians with Type 2 diabetes. J Diabetes Complicat. 2009;23:102-11.

15. Kordonouri O, James RW, Bennetts B, et al. Modulation by blood glucose levels of activity and concentration of paraoxonase in young patients with type 1 diabetes mellitus. Metabolism. 2001;50:657-60.

16. El-Lebedy D, Kafoury M, Abd-El Haleem D, Ibrahim A, Awadallah E, Ashmawy I. Paraoxonase-1 gene Q192R and L55M polymorphisms and risk of cardiovascular disease in Egyptian patients with type 2 diabetes mellitus. J Diabetes Metab Disord. 2014;13:124.

17. Letellier C, Durou MR, Jouanolle AM, Le Gall JY, Poirier JY, Ruelland A. Serum paraoxonase activity and paraoxonase gene polymorphism in type 2 diabetic patients with or without vascular complications. Diabetes Metab. 2002;28:297-304.

18. Hofer SE, Bennetts B, Chan AK, et al. Association between PON 1 polymorphisms, PON activity and diabetes complications. J Diabetes Complicat. 2006;20:322-8.

19. Zheng Y, Lei Y, Gong J, Zhong X, Pan T. Association of paraoxonase 1 gene polymorphisms with retinopathy in type 2 diabetes mellitus. Acta Universitatis Medicinalis Anhui. 2012;47:1206-8.

20. Chen X, Pan S, Zeng J. Relationship between PON1, PON2 polymorphism and type 2 diabetic nephropathy. Med J Chin. 2011;22:153-6.

21. Shi $\mathrm{G}, \mathrm{Wu}$ N. Relationship between paraoxonase 1 genetic polymorphism with type 2 diabetic nephropathy. Clin Med China. 2007;23:134-6.

22. Zhang Z, Li J, Zhang C. PON1 gene polymorphism in NIDDM patients with cerebral infarction. Acta Academinae Medicine Qingdao Universitatis. 2004;40:336-8.

23. Li S, Li J, Wei G, Ji Q, Wang P. Study on the relationship between paraoxonase 1 and type 2 diabetes mellitus complicated by coronary artery disease. Tianjin Med J. 2004;32:273-6.

24. Ren T, Xiang K, Liu L. An association study of diabetic neruopathy with polymorphisms of eNOS, 
PON1, RAGE and ALR2 genes. Shanghai Med J. 2003;26:24-7.

25. Pu X, Zeng F, Zeng Z, Liu W, Ke X. Study on relationship between paraoxonase 1 and type 2 diabetes mellitus in the elderly. Chin J Geriatr. $2003 ; 22: 466-8$.

26. Wang Y, Chang Z. The relationship between PON1 192 Gln/Arg gene polymorphism and type 2 diabetes mellitus complicated with coronary disease. Guangdong Med J. 2003;24:598-600.

27. Qi L, Lu Z, Dong Y. The association of paraoxonase$1192 \mathrm{Gln} /$ Arg and paraoxonase-2 311 Cys/Ser gene polymorphisms with macrovascular disease in type 2 diabetes mellitus patients. Chin J Arterioscler. 2007;15:703-7.

28. Ma R, Yan S, Yu H, Zhao S, Wang Y, Miao Z. The association of paraoxonase $192 \mathrm{Gln} /$ Arg gene polymorphism with coronary heart disease with type 2 diabetes mellitus. Chin J Diabetes. 2003;11:29-33.

29. Qian Q, Qian S, Wang S. Association of paraoxonase 1 arylersterase gene polymorphism in patients with type 2 diabetes and coronary heart disease. J Clin Cardiol (China). 2003;19:606-9.

30. Sun Y, Sun S, Zuo J, Lin Y, Kan Y, Shao H. Polymorphism of paraoxonase in diabetic nephropathies. J Jilin Univ (Med Ed). 2005;31:598-601.
31. Shao H, Zhang Y. The association of paraoxonase gene polymorphism and coronary disease in patients with type 2 diabetes mellitus. Shanxi Med J. 2006;35:1432-3.

32. Shao Z, Li J, Wang X. Analysis of paraoxonase 1 gene polymorphisms in type 2 diabetic patients with coronary artery disease. Chin J Cardiovasc Med. 2014;19:426-9.

33. James RW, Deakin SP. The importance of highdensity lipoproteins for paraoxonase-1 secretion, stability, and activity. Free Radic Biol Med. 2004;37:1986-94.

34. Leviev I, Deakin S, James RW. Decreased stability of the M54 isoform of paraoxonase as a contributory factor to variations in human serum paraoxonase concentrations. J Lipid Res. 2001;42:528-35.

35. Chen L, Lu W, Fang L, et al. Association between L55M polymorphism in paraoxonase 1 and cancer risk: a meta-analysis based on 21 studies. Onco Targets Ther. 2016;9:1151-8.

36. Wang J, Yang MM, Rong SS, Ng TK, Li YB, Liu XM. Association of paraoxonase gene polymorphisms with diabetic nephropathy and retinopathy. Mol Med Rep. 2013;8:1845-51. 